\title{
AN EXTRAGRADIENT-LIKE PARALLEL METHOD FOR PSEUDOMONOTONE EQUILIBRIUM PROBLEMS AND SEMIGROUP OF NONEXPANSIVE MAPPINGS
}

\author{
L.Q. THUY, C.-F. WEN, J.-C. YAO, AND T. N. HAI
}

Received 11 October, 2016

\begin{abstract}
In this paper we propose a parallel iterative hybrid methods for finding a common element of the solution sets of a finite family of pseudomonotone equilibrium problems and the fixed points set of a semigroup-nonexpensive mappings in Hilbert spaces. Under mild conditions, we obtain the strong convergence of the proposed iterative process. Some numerical experiments are given to verify the efficiency the proposed algorithm.
\end{abstract}

2010 Mathematics Subject Classification: 65K10; 90C25; 90C33; 65Y05; 68W10

Keywords: equilibrium problems, fixed point problems, pseudomonotonicity, nonexpansive mappings, nonexpansive semigroup, parallel computation

\section{INTRODUCTION}

Throughout the paper we suppose that $\mathscr{H}$ is a real Hilbert space with inner product $\langle\cdot, \cdot\rangle$ and the associated norm $\|\cdot\|, C$ is a closed convex subset in $\mathscr{H}$ and that $f_{i}$ : $C \times C \rightarrow \mathbb{R}, i=1,2, \ldots, N, T(s): C \rightarrow C, s \geq 0$. Conditions for $f_{i}, i=1,2, \ldots, N$ and $T(s)$ will be detailed later. We are interested in a solution method for the system defined as

$$
\begin{array}{ll}
\text { Find } x^{*} \in C: \quad & f_{i}\left(x^{*}, y\right) \geq 0 \quad \forall y \in C, i=1,2, \ldots, N, \\
& x^{*}=T(s)\left(x^{*}\right) \quad \forall s \geq 0 .
\end{array}
$$

The problem (1.1) can be considered as a system of equilibrium problems, which was first introduced by Blum and Oettli in [2]: Given $f: C \times C \rightarrow \mathcal{R}$,

$$
\text { find } x^{*} \in C \text { such that } f\left(x^{*}, y\right) \geq 0 \quad \forall y \in C \text {. }
$$

The equilibrium problems play an important role in optimization and nonlinear analysis. It is well known that many problems, such as variational inequalities, Nash equilibrium problems, saddle point problems, complementarity problems can be formulated as special cases of equilibrium problems. There are different methods for 
solving (1.3), see, for example, [1-4], [5], [8], [12-16], [20], [21,22], and the references cited therein. Among them, we are interested in Extragradient method introduced in $[9,20]$ due to its simplicity and efficiency:

$$
\left\{\begin{array}{l}
x_{0} \in C, \\
y_{n}=\underset{y \in C}{\operatorname{argmin}}\left\{\lambda f\left(x_{n}, y\right)+\frac{1}{2}\left\|y-x_{n}\right\|^{2}\right\}, \\
x_{n+1}=\underset{y \in C}{\operatorname{argmin}}\left\{\lambda f\left(y_{n}, y\right)+\frac{1}{2}\left\|y-x_{n}\right\|^{2}\right\} .
\end{array}\right.
$$

Under assumptions that the bifunctions $f$ is pseudomonone and Lipschitz-type continuous, the authors proved that the sequence $\left\{x_{n}\right\}$ generated by (1.4) weakly converges to a solution of (1.3).

It is well known that the problems of finding common fixed points of nonexpansive mappings and of nonexpansive semigroups is an important problem in fixed point theory and applications; in particular, in image recovery, convex feasibility problem, and signal processing problem (see e.g. [7], [14]). Iterative approximation methods for these problems in Hilbert or Banach spaces have been studied extensively by many authors; see, for example, [6], [17], [23], [24, 25], and the references therein. Finding a common element of the set of fixed points of nonexpansive mappings or a semigroup of nonexpansive mappings and the set of solutions to a equilibrium problem has been studied extensively in the literature; see, for example, [4], [5], [12], [17], [18], [21,22], [25] and the references therein. The common approach in these papers is to use a proximal point algorithm for handling the equilibrium problem. For monotone equilibrium problems the subproblems needed to solve in the proximal point method are strongly monotone, and therefore they have a unique solution that can be approximated by available methods. However, for pseudomonone problems the subproblems, in general, may have nonconvex solution set due to the fact that the regularized bifunctions do not inherit any pseudomonotoniciy property from the original one.

In this article, motivated by $[4,11]$ and inspired by $[9,20]$, we propose a parallel iterative method for finding a common element of the solution sets of a finite family of pseudomonotone equilibrium problems and the set of fixed points of a nonexpansive semigroup in Hilbert spaces. The main point here is that we combine a parallel splitting-up technique and the extragradient procedure rather than a proximal point algorithm for dealing with a finite family of pseudomonotone equilibrium problems and Mann's iterative algorithms for finding fixed points of nonexpansive mappings. We obtain the strong convergence of iterative processes.

The paper is organized as follows: In Section 2, we collect some definitions and results needed for further investigation. We describe a novel parallel hybrid iterative 
method in the Section 3. The convergence analysis for the proposed method is detailed in Section 4. Some special cases and illustrative examples are provided in the last section.

\section{PRELIMINARIES}

In this section, we recall some definitions and results that will be used in the sequel. In what follows by $x_{n} \rightarrow x$ we mean that the sequence $\left\{x_{n}\right\}$ converges to $x$ in the weak topology. Let $C$ be a nonempty closed convex of a Hilbert space $H$. We recall that mapping $T: C \rightarrow C$ is said to be nonexpansive on $C$ if

$$
\|T x-T y\| \leq\|x-y\| \text { for al } x, y \in C .
$$

Let $F(T)$ denote the set of fixed points of $T$. A family $\left\{T(s): s \in \mathbb{R}_{+}\right\}$of mappings from $C$ into itself is called a nonexpansive semigroup on $C$ if it satisfies the following conditions:

(i) for each $s \in \mathbb{R}_{+}, T(s)$ is a nonexpansive mapping on $C$;

(ii) $T(0) x=x$ for all $x \in C$;

(iii) $T\left(s_{1}+s_{2}\right)=T\left(s_{1}\right) \circ T\left(s_{2}\right)$ for all $s_{1}, s_{2} \in \mathbb{R}_{+}$;

(iv) for each $x \in C$, the mapping $T(\cdot) x$ from $\mathbb{R}_{+}$into $C$ is continuous.

Let $\mathcal{F}=\bigcap_{s \geq 0} F(T(s))$ be the set of all common fixed points of $\left\{T(s): s \in \mathbb{R}_{+}\right\}$. We know that $\widetilde{F}$ is nonempty if $C$ is bounded (see [3]).

We begin with the following properties of nonexpansive mappings.

Lemma 1 ([10]). Let $C$ be a closed convex subset of a Hilbert space $\mathcal{H}$ and let $S: C \rightarrow C$ be a nonexpansive mapping such that $F(S) \neq \varnothing$. If a sequence $\left\{x_{n}\right\} \subset C$ such that $x_{n} \rightarrow z$ and $x_{n}-S x_{n} \rightarrow 0$, then $z=S z$.

Lemma 2 ([23]). Let $C$ be a nonempty bounded closed convex subset of $\mathscr{H}$ and let $\left\{T(s): s \in \mathbb{R}_{+}\right\}$be a nonexpansive semigroup on $C$. Then, for any $h \geq 0$

$$
\lim _{s \rightarrow \infty} \sup _{y \in C}\left\|T(h)\left(\frac{1}{s} \int_{0}^{s} T(t) y d t\right)-\frac{1}{s} \int_{0}^{s} T(t) y d t\right\|=0
$$

Since $C$ is a nonempty closed and convex subset of $H$, for every $x \in H$, there exists a unique element $P_{C} x$, defined by

$$
P_{C} x=\arg \min \{\|y-x\|: y \in C\} .
$$

The mapping $P_{C}: H \rightarrow C$ is called the metric (orthogonal) projection of $H$ onto $C$. It is also known that $P_{C}$ is firmly nonexpansive, or 1-inverse strongly monotone (1-ism), i.e.,

$$
\left\langle P_{C} x-P_{C} y, x-y\right\rangle \geq\left\|P_{C} x-P_{C} y\right\|^{2} \forall x, y \in H .
$$

Besides, we have

$$
\left\|x-P_{C} y\right\|^{2}+\left\|P_{C} y-y\right\|^{2} \leq\|x-y\|^{2} \quad \forall x \in C, \forall y \in H .
$$


Moreover, $z=P_{C} x$ if only if

$$
\langle x-z, z-y\rangle \geq 0 \quad \text { for all } y \in C .
$$

The bifunction $f: C \times C \rightarrow \mathbb{R}$ is called monotone on $C$ if

$$
f(x, y)+f(y, x) \leq 0 \text { for all } x, y \in C ;
$$

pseudomonotone on $C$ if

$$
f(x, y) \geq 0 \Rightarrow f(y, x) \leq 0 \text { for all } x, y \in C .
$$

It is obvious that any monotone bifunction is a pseudomonotone one, but not vice versa.

Throughout this paper we consider bifunctions with the following properties:

(C1) $f$ is pseudomonotone, i.e., for all $x, y \in C$,

$$
f(x, y) \geq 0 \Rightarrow f(y, x) \leq 0 ;
$$

(C2) $f$ is Lipschitz-type continuous, i.e., there exist two positive constants $c_{1}, c_{2}$ such that

$$
f(x, y)+f(y, z) \geq f(x, z)-c_{1}\|x-y\|^{2}-c_{2}\|y-z\|^{2} \text { for all } x, y, z \in C ;
$$

(C3) $f$ is weakly continuous on $C \times C$;

(C4) $f(x,$.$) is convex, subdifferentiable on C$ and $f(x, x)=0$ for every $x \in C$.

The following statements will be needed in the next section.

Lemma 3 ([1]). If the bifunction $f$ satisfies Assumptions $(C 1)-(C 4)$, then the solution set of equilibrium problems:

$$
\text { Find } x^{*} \in C: f\left(x^{*}, y\right) \geq 0 \text { for all } y \in C
$$

is weakly closed and convex.

Lemma 4 ([8]). Let $C$ be a convex subset of a real Hilbert space $H$ and $g: C \rightarrow$ $\mathbb{R}$ be a convex and subdifferentiable function on $C$. Then, $x^{*}$ is a solution to the following convex problem

$$
\min \{g(x): x \in C\}
$$

if only if $0 \in \partial g\left(x^{*}\right)+N_{C}\left(x^{*}\right)$, where $\partial g($.$) denotes the subdifferential of g$ and $N_{C}\left(x^{*}\right)$ is the normal cone of $C$ at $x^{*}$.

It is also known that $\mathscr{H}$ satisfies Opial's condition. See the following definition in [19].

Definition 1 ([19]). A Banach space $X$ is said to satisfy Opial's condition if whenever $\left\{x_{n}\right\}$ is a sequence in $X$ which converges weakly to $x$, as $k \rightarrow \infty$, then

$$
\lim _{n \rightarrow \infty} \sup \left\|x_{n}-x\right\|<\lim _{n \rightarrow \infty} \sup \left\|x_{n}-y\right\| \quad \forall y \in X \text {, with } x \neq y .
$$




\section{MAin Results}

In this section, based on the hybrid method in mathematical programming, projection, extragradient method and Mann's iteration, we propose a parallel iterative method for finding a common element of the solution sets of a finite family of equilibrium problems with pseudomonotone bifunctions $\left\{f_{i}\right\}_{i=1}^{N}$ and the set of fixed points of nonexpansive semigroup mappings $\{T(s): s \geq 0\}$ in a Hilbert space $\mathscr{H}$.

We denote by $\operatorname{Sol}\left(C, f_{i}\right)$ the set of $(1.1), i=1,2, \ldots, N,$. In what follows, we assume that the solution set

$$
\Omega=\mathscr{F} \bigcap\left(\bigcap_{i=1}^{N} \operatorname{Sol}\left(C, f_{i}\right)\right)
$$

is nonempty and each bifunction $f_{i},(i=1, \ldots, N)$ satisfies all the conditions $(C 1)-$ (C4). Observe that we can choose the same Lipschitz coefficients $\left\{c_{1}, c_{2}\right\}$ for all bifunctions $f_{i}, i=1,2, \ldots, N$. Indeed, condition $(C 2)$ implies that

$f_{i}(x, z)-f_{i}(x, y)-f_{i}(y, z) \leq c_{1 i}\|x-y\|^{2}+c_{2 i}\|y-z\|^{2} \leq c_{1}\|x-y\|^{2}+c_{2}\|y-z\|^{2}$, where $c_{1}=\max _{1 \leq i \leq N} c_{1 i}$ and $c_{2}=\max _{1 \leq i \leq N} c_{2 i}$. Hence,

$$
f_{i}(x, y)+f_{i}(y, z) \geq f_{i}(x, z)-c_{1}\|x-y\|^{2}-c_{2}\|y-z\|^{2} .
$$

Further, since $\Omega \neq \varnothing$, by Lemma 3, the sets $\operatorname{Sol}\left(C, f_{i}\right), i=1, \ldots, N$ are nonempty, closed and convex, hence the solution set $\Omega$ is a nonempty closed and convex subset of $C$. Thus given any fixed element $x_{0} \in C$ there exists a unique element $\bar{x}:=P_{\Omega} x_{0}$.

Algorithm 1. Choose positive number $0<\rho<\min \left(\frac{1}{2 c_{1}}, \frac{1}{2 c_{2}}\right)$ and the positive sequences $\left\{\mu_{n}\right\} \subset[a, 1]$ for some $a \in(0,1)$.

Seek a starting point $x_{0} \in C$ and set $n:=0$.

Step 1.

- Solve the strongly convex programs

$$
\begin{aligned}
& y_{n}^{i}=\operatorname{argmin}\left\{\rho f_{i}\left(x_{n}, y\right)+\frac{1}{2}\left\|x_{n}-y\right\|^{2}: y \in C\right\}, \\
& z_{n}^{i}=\operatorname{argmin}\left\{\rho f_{i}\left(y_{n}^{i}, y\right)+\frac{1}{2}\left\|x_{n}-y\right\|^{2}: y \in C\right\}, i=1, \ldots, N ;
\end{aligned}
$$

- Find positive integer

$$
i_{n}=\underset{1 \leq i \leq N}{\operatorname{argmax}}\left\{\left\|z_{n}^{i}-x_{n}\right\|\right\},
$$

and set $z_{n}:=z_{n}^{i_{n}}$

Step 2. Compute $u_{n}=\left(1-\mu_{n}\right) x_{n}+\mu_{n} T_{n} z_{n}$, where $T_{n}$ is defined as

$$
T_{n} x:=\frac{1}{s_{n}} \int_{0}^{s_{n}} T(s) x d s, \forall x \in C \text { with } \lim _{n \rightarrow+\infty} s_{n}=+\infty ;
$$




$$
\begin{aligned}
& x_{n+1}=P_{\left(H_{n} \cap W_{n}\right)} x_{0}, \text { where } \\
& \qquad H_{n}=\left\{z \in \mathscr{H}:\left\|u_{n}-z\right\| \leq\left\|x_{n}-z\right\|\right\}, \\
& W_{n}=\left\{z \in \mathcal{H}:\left\langle x_{n}-z, x_{0}-x_{n}\right\rangle \geq 0\right\} .
\end{aligned}
$$

Increase $n$ by 1 and go back to Step 1 .

We now state and prove the convergence of the proposed iteration method.

\section{Convergence Results}

In this section, we show the strong convergence of the sequences $\left\{x_{n}\right\}$ and $\left\{u_{n}\right\}$ defined by Algorithm 1 to the common element in a real Hilbert space.

For establishing the strong convergence of $\left\{x_{n}\right\}$ and $\left\{u_{n}\right\}$ in Algorithm 1, we need the following result (see [20]).

Lemma 5. Suppose that $x^{*} \in \operatorname{Sol}\left(C, f_{i}\right)$ and $x_{n}, y_{n}^{i}, z_{n}^{i}, i=1, \ldots, N$, are as in Step 1 of Algorithm 1. Then

$$
\left\|z_{n}^{i}-x^{*}\right\|^{2} \leq\left\|x_{n}-x^{*}\right\|^{2}-\left(1-2 \rho c_{1}\right)\left\|y_{n}^{i}-x_{n}\right\|^{2}-\left(1-2 \rho c_{2}\right)\left\|y_{n}^{i}-z_{n}^{i}\right\|^{2} .
$$

Theorem 1. Let $C$ be a nonempty closed convex subset in a real Hilbert space $\mathscr{H}$, $\left\{T(s): s \in \mathbb{R}_{+}\right\}$be a nonexpansive semigroup on $C$, $f_{i}$ be a bifunction from $C \times C$ to $\mathbb{R}$ satisfying conditions $\left(C_{1}\right)-\left(C_{4}\right)$. Suppose that $\Omega \neq \varnothing$. Let $\left\{x_{n}\right\}$ and $\left\{u_{n}\right\}$ be sequences generated by the Algorithm 1 , where $\left\{\mu_{n}\right\} \subset[a, 1]$ for some $a \in(0,1)$. Then, $\left\{x_{n}\right\}$ and $\left\{u_{n}\right\}$ converge strongly to an element $p^{*}=P_{\Omega} x_{0}$.

Proof. It is obvious that $H_{n}$ and $W_{n}$ are closed and convex for every $n \geq 0$. So that the $\left\{x_{n}\right\}$ is well defined for every $n \geq 0$. Moreover, it is easy seen that $T_{n}$ is nonexpensive for all $n \geq 0$.

Now we divide the proof into several steps.

Step 1. Claim that $\Omega \subset H_{n} \cap W_{n}$ for every $n \geq 0$.

Indeed, for each $x^{*} \in \Omega$, by Lemma 5 , we have

$$
\left\|z_{n}^{i}-x^{*}\right\| \leq\left\|x_{n}-x^{*}\right\| \text { for all } n \geq 0 .
$$

From the definition of $i_{n}$, we have

$$
\left\|z_{n}-x^{*}\right\| \leq\left\|x_{n}-x^{*}\right\| \text { for all } n \geq 0 .
$$

From the convexity of $\|\cdot\|^{2}$, the nonexpansiveness of $T_{n}$ and (4.1) it follows that

$$
\begin{aligned}
\left\|u_{n}-x^{*}\right\|^{2} & =\left\|\left(1-\mu_{n}\right)\left(x_{n}-x^{*}\right)+\mu_{n}\left(T_{n} z_{n}-x^{*}\right)\right\|^{2} \\
& \leq\left(1-\mu_{n}\right)\left\|x_{n}-x^{*}\right\|^{2}+\mu_{n}\left\|T_{n} z_{n}-T_{n} x^{*}\right\|^{2} \\
& \leq\left(1-\mu_{n}\right)\left\|x_{n}-x^{*}\right\|^{2}+\mu_{n}\left\|z_{n}-x^{*}\right\|^{2} \\
& \leq\left(1-\mu_{n}\right)\left\|x_{n}-x^{*}\right\|^{2}+\mu_{n}\left\|x_{n}-x^{*}\right\|^{2} \\
& =\left\|x_{n}-x^{*}\right\|^{2} \quad \forall n \geq 0,
\end{aligned}
$$


which implies $x^{*} \in H_{n}$. Hence $\Omega \subset H_{n}$ for all $n \geq 0$.

Next we show $\Omega \subset W_{n}$ for all $n \geq 0$. Indeed, when $n=0$, we have $x_{0} \in C$ and $W_{0}=H$. Consequently, $\Omega \subset H_{0} \cap W_{0}$. By induction, suppose $\Omega \subset H_{m} \cap W_{m}$ for some $m \geq 0$. We have to prove that $\Omega \subset H_{m+1} \cap W_{m+1}$. Since $x_{m+1}=P_{H_{m} \cap W_{m}} x_{0}$, by (2.3), for every $z \in \Omega \subset H_{m} \cap W_{m}$, it holds that

$$
\left\langle x_{m+1}-z, x_{0}-x_{m+1}\right\rangle \geq 0,
$$

which means that $z \in W_{m+1}$. Note that $\Omega \subset H_{n}$ for all $n \geq 0$, we can conclude that $\Omega \subset H_{n} \cap W_{n}$ for all $n \geq 0$.

Step 2. Claim that for all $i=1, \ldots, N$, we have

$$
\lim _{n \rightarrow \infty}\left\|x_{n+1}-x_{n}\right\|=\lim _{n \rightarrow \infty}\left\|x_{n}-u_{n}\right\|=\lim _{n \rightarrow \infty}\left\|x_{n}-z_{n}^{i}\right\|=\lim _{n \rightarrow \infty}\left\|x_{n}-y_{n}^{i}\right\|=0 .
$$

Indeed, from $x_{n}=P_{W_{n}} x_{0}$ and (2.2), it follows that, for every $u \in \Omega \subset W_{n}$, we get

$$
\left\|x_{n}-x_{0}\right\|^{2} \leq\left\|u-x_{0}\right\|^{2}-\left\|u-x_{n}\right\|^{2} \leq\left\|u-x_{0}\right\|^{2} .
$$

This implies that the sequence $\left\{x_{n}\right\}$ is bounded. From (4.2) and (4.1), it follows that the sequences $\left\{u_{n}\right\}$ and $\left\{z_{n}\right\}$ are also bounded.

Observing that $x_{n+1}=P_{H_{n} \cap W_{n}} x_{0} \in W_{n}, x_{n}=P_{W_{n}} x_{0}$, from (2.2) we have

$$
\left\|x_{n}-x_{0}\right\|^{2} \leq\left\|x_{n+1}-x_{0}\right\|^{2}-\left\|x_{n+1}-x_{n}\right\|^{2} \leq\left\|x_{n+1}-x_{0}\right\|^{2} .
$$

Thus, the sequence $\left\{\left\|x_{n}-x_{0}\right\|\right\}$ is nondecreasing, hence there exists the limit of the sequence $\left\{\left\|x_{n}-x_{0}\right\|\right\}$. From (4.4) we obtain

$$
\left\|x_{n+1}-x_{n}\right\|^{2} \leq\left\|x_{n+1}-x_{0}\right\|^{2}-\left\|x_{n}-x_{0}\right\|^{2} \text {. }
$$

Letting $n \rightarrow \infty$, we find

$$
\lim _{n \rightarrow \infty}\left\|x_{n+1}-x_{n}\right\|=0 .
$$

Since $x_{n+1} \in H_{n}$, it follows that $\left\|u_{n}-x_{n+1}\right\| \leq\left\|x_{n+1}-x_{n}\right\|$. Thus

$$
\left\|u_{n}-x_{n}\right\| \leq\left\|u_{n}-x_{n+1}\right\|+\left\|x_{n+1}-x_{n}\right\| \leq 2\left\|x_{n+1}-x_{n}\right\| .
$$

The last inequality together with (4.5) implies that

$$
\lim _{n \rightarrow \infty}\left\|u_{n}-x_{n}\right\|=0
$$

Moreover, from (4.2), Lemma 5 and the definition of $i_{n}$ for any fixed $x^{*} \in \Omega$, we have

$$
\begin{aligned}
\left\|u_{n}-x^{*}\right\|^{2} & \leq\left(1-\mu_{n}\right)\left\|x_{n}-x^{*}\right\|^{2}+\mu_{n}\left\|z_{n}-x^{*}\right\|^{2} \\
& \leq\left\|x_{n}-x^{*}\right\|^{2}-\mu_{n}\left[\left(1-2 \rho c_{1}\right)\left\|y_{n}^{i_{n}}-x_{n}\right\|^{2}+\left(1-2 \rho c_{2}\right)\left\|y_{n}^{i_{n}}-z_{n}\right\|^{2}\right] .
\end{aligned}
$$

Therefore

$$
\begin{aligned}
& a\left[\left(1-2 \rho c_{1}\right)\left\|y_{n}^{i_{n}}-x_{n}\right\|^{2}+\left(1-2 \rho c_{2}\right)\left\|y_{n}^{i_{n}}-z_{n}\right\|^{2}\right] \\
& \leq \mu_{n}\left[\left(1-2 \rho c_{1}\right)\left\|y_{n}^{i_{n}}-x_{n}\right\|^{2}+\left(1-2 \rho c_{2}\right)\left\|y_{n}^{i_{n}}-z_{n}\right\|^{2}\right]
\end{aligned}
$$




$$
\begin{aligned}
& \leq\left\|x_{n}-x^{*}\right\|^{2}-\left\|u_{n}-x^{*}\right\|^{2} \\
& =\left(\left\|x_{n}-x^{*}\right\|-\left\|u_{n}-x^{*}\right\|\right)\left(\left\|x_{n}-x^{*}\right\|+\left\|u_{n}-x^{*}\right\|\right) \\
& \leq\left\|x_{n}-u_{n}\right\|\left(\left\|x_{n}-x^{*}\right\|+\left\|u_{n}-x^{*}\right\|\right) .
\end{aligned}
$$

Using the last inequality together with (4.6) and taking into account the boundedness of two sequences $\left\{u_{n}\right\}$ and $\left\{x_{n}\right\}$ as well as the conditions of $\left\{\mu_{n}\right\}, \rho$, we come to the relations

$$
\lim _{n \rightarrow \infty}\left\|y_{n}^{i_{n}}-x_{n}\right\|=\lim _{n \rightarrow \infty}\left\|y_{n}^{i_{n}}-z_{n}\right\|=0 .
$$

From $\left\|z_{n}-x_{n}\right\| \leq\left\|z_{n}-y_{n}^{i_{n}}\right\|+\left\|y_{n}^{i_{n}}-x_{n}\right\|$ and (4.8), we obtain

$$
\lim _{n \rightarrow \infty}\left\|z_{n}-x_{n}\right\|=0 \text {. }
$$

By the definition of $i_{n}$, we get

$$
\lim _{n \rightarrow \infty}\left\|z_{n}^{i}-x_{n}\right\|=0
$$

for all $i=1, \ldots, N$. From Lemma 5 and (4.10), we obtain

$$
\lim _{n \rightarrow \infty}\left\|y_{n}^{i}-x_{n}\right\|=0
$$

for all $i=1, \ldots, N$.

Since $\left\{x_{n}\right\}$ is bounded, there exists a subsequence $\left\{x_{n_{k}}\right\}$ of $\left\{x_{n}\right\}$ converging weakly to some element $p$.

Step 3. Claim that $p \in \Omega$.

From (4.6) and (4.9), we obtain also that $\left\{u_{n_{k}}\right\}$ and $\left\{z_{n_{k}}\right\}$ converges weakly to $p$. Since $\left\{u_{n}\right\} \subset C$ and $C$ is a closed convex subset in $\mathscr{H}$, we have $p \in C$.

Now, we prove that $p \in \Omega$. To this end, first we show that $p \in \underset{i=1}{N} \operatorname{Sol}\left(C, f_{i}\right)$. Noting that

$$
y_{n}^{i}=\operatorname{argmin}\left\{\rho f_{i}\left(x_{n}, y\right)+\frac{1}{2}\left\|x_{n}-y\right\|^{2}: y \in C\right\},
$$

by Lemma 4, we obtain

$$
0 \in \partial_{2}\left\{\rho f_{i}\left(x_{n}, y\right)+\frac{1}{2}\left\|x_{n}-y\right\|^{2}\right\}\left(y_{n}^{i}\right)+N_{C}\left(y_{n}^{i}\right) .
$$

Therefore, there exists $w \in \partial_{2} f_{i}\left(x_{n}, y_{n}^{i}\right)$ and $\bar{w} \in N_{C}\left(y_{n}^{i}\right)$ such that

$$
\rho w+x_{n}-y_{n}^{i}+\bar{w}=0 .
$$

Since $\bar{w} \in N_{C}\left(y_{n}^{i}\right),\left\langle\bar{w}, y-y_{n}^{i}\right\rangle \leq 0$ for all $y \in C$. This together with (4.11) implies that

$$
\rho\left\langle w, y-y_{n}^{i}\right\rangle \geq\left\langle y_{n}^{i}-x_{n}, y-y_{n}^{i}\right\rangle
$$


for all $y \in C$. Since $w \in \partial_{2} f_{i}\left(x_{n}, y_{n}^{i}\right)$,

$$
f_{i}\left(x_{n}, y\right)-f_{i}\left(x_{n}, y_{n}^{i}\right) \geq\left\langle w, y-y_{n}^{i}\right\rangle \text { for all } y \in C .
$$

From (4.12) and (4.13), we get

$$
\rho\left(f_{i}\left(x_{n}, y\right)-f_{i}\left(x_{n}, y_{n}^{i}\right)\right) \geq\left\langle y_{n}^{i}-x_{n}, y-y_{n}^{i}\right\rangle \text { for all } y \in C .
$$

Since $x_{n_{k}} \rightarrow p$ and $\left\|x_{n}-y_{n}^{i}\right\| \rightarrow 0$ as $n \rightarrow \infty$, we have $y_{n_{k}}^{i} \rightarrow p$. Letting $n=n_{k}$ in (4.14), passing to the limit as $k \rightarrow \infty$ and using assumptions (C3), we conclude that $f_{i}(p, y) \geq 0$ for all $y \in C, i=1,2, \ldots, N$. Thus, $p \in \bigcap_{i=1}^{N} \operatorname{Sol}\left(C, f_{i}\right)$.

Now, we prove that $p=T(h) p$ for all $h>0$. First, we obtain from Step 3 of the algorithm that

$$
\begin{aligned}
a\left\|u_{n}-T_{n} u_{n}\right\| & \leq \mu_{n}\left\|u_{n}-T_{n} u_{n}\right\| \\
& \leq \mu_{n}\left(\left\|u_{n}-T_{n} z_{n}\right\|+\left\|T_{n} z_{n}-T_{n} u_{n}\right\|\right) \\
& \leq\left\|\mu_{n} u_{n}-\mu_{n} T_{n} z_{n}\right\|+\mu_{n}\left\|T_{n} z_{n}-T_{n} u_{n}\right\| \\
& =\left\|\mu_{n} u_{n}+\left(1-\mu_{n}\right) x_{n}-u_{n}\right\|+\mu_{n}\left\|T_{n} z_{n}-T_{n} u_{n}\right\| \\
& \leq\left(1-\mu_{n}\right)\left\|x_{n}-u_{n}\right\|+\mu_{n}\left\|z_{n}-u_{n}\right\| \\
& \leq\left\|u_{n}-x_{n}\right\|+\mu_{n}\left\|z_{n}-x_{n}\right\|
\end{aligned}
$$

Taking into account $\left\|u_{n}-x_{n}\right\| \rightarrow 0$ and $\left\|z_{n}-x_{n}\right\| \rightarrow 0$, it follows that

$$
\lim _{n \rightarrow \infty}\left\|u_{n}-T_{n} u_{n}\right\|=0 .
$$

Note that

$$
\begin{aligned}
\left\|T(h) u_{n}-u_{n}\right\| & \leq\left\|T(h) u_{n}-T(h)\left(\frac{1}{s_{n}} \int_{0}^{s_{n}} T(s) u_{n} d s\right)\right\| \\
& +\left\|T(h)\left(\frac{1}{s_{n}} \int_{0}^{s_{n}} T(s) u_{n} d s\right)-\frac{1}{s_{n}} \int_{0}^{s_{n}} T(s) u_{n} d s\right\| \\
& +\left\|\frac{1}{s_{n}} \int_{0}^{s_{n}} T(s) u_{n} d s-u_{n}\right\| \\
& \leq 2\left\|\frac{1}{s_{n}} \int_{0}^{s_{n}} T(s) u_{n} d s-u_{n}\right\| \\
& +\left\|T(h)\left(\frac{1}{s_{n}} \int_{0}^{s_{n}} T(s) u_{n} d s\right)-\frac{1}{s_{n}} \int_{0}^{s_{n}} T(s) u_{n} d s\right\| .
\end{aligned}
$$

Since the sequence $\left\{u_{n}\right\}$ is bounded, we can apply Lemma 2 to get

$$
\lim _{n \rightarrow \infty}\left\|T(h)\left(\frac{1}{s_{n}} \int_{0}^{s_{n}} T(s) u_{n} d s\right)-\frac{1}{s_{n}} \int_{0}^{s_{n}} T(s) u_{n} d s\right\|=0,
$$


for every $h \in(0, \infty)$ and therefore, by (4.16), (4.17) and (4.18), we obtain

$$
\lim _{n \rightarrow \infty}\left\|T(h) u_{n}-u_{n}\right\|=0
$$

for each $h>0$ from which we have by Lemma 1 that $p$ is a fixed point of $T(h)$ for all $h>0$. Hence $p \in \mathcal{F}$.

Step 4. The sequence $\left\{x_{n}\right\}$ converges strongly to $p^{*}:=P_{\Omega} x_{0}$.

Indeed, from $p^{*} \in \Omega$ and (4.3), we obtain

$$
\left\|x_{n}-x_{0}\right\| \leq\left\|p^{*}-x_{0}\right\| \forall n \geq 0 .
$$

The last inequality together with $x_{n_{k}} \rightarrow p$ and the weak lower semicontinuity of the norm $\|$.$\| imply that$

$$
\left\|p-x_{0}\right\| \leq \lim \inf _{k \rightarrow \infty}\left\|x_{n_{k}}-x_{0}\right\| \leq \lim \sup _{k \rightarrow \infty}\left\|x_{n_{k}}-x_{0}\right\| \leq\left\|p^{*}-x_{0}\right\| .
$$

On the other hand, since $p^{*}:=P_{\Omega} x_{0}$, we have $\left\|p-x_{0}\right\| \geq\left\|p^{*}-x_{0}\right\|$. Hence, $p^{*}=p$ and $\lim _{k \rightarrow \infty}\left\|x_{n_{k}}-x_{0}\right\|=\left\|p^{*}-x_{0}\right\|$. Taking into account $x_{n_{k}} \rightarrow p^{*}$, we have $x_{n_{k}} \rightarrow p^{*}$. Finally, suppose that $\left\{x_{n_{j}}\right\}$ is an another weakly convergent subsequence of $\left\{x_{n}\right\}$. By a similar argument as above, we conclude that $\left\{x_{n_{j}}\right\}$ converges strongly to $p^{*}:=P_{\Omega} x_{0}$. Therefore, the sequence $\left\{x_{n}\right\}$ generated by the Algorithm 1 converges strongly to $P_{\Omega} x_{0}$. Then the strong convergence of the sequences $\left\{u^{n}\right\}$ to $p$ is followed from (4.6). The proof is now completed.

\section{SPECIAL CASES AND ILlustrative EXAMPLES}

If $N=1$, Algorithm 1 reduces to the following one for finding a common element in the solution set of pseudomonotone equilibrium problems and the set of the fixed points of a nonexpansive semigroup in Hilbert spaces.

Corollary 1. Let $C$ be a nonempty closed convex subset in a real Hilbert space $\mathscr{H},\left\{T(s): s \in \mathbb{R}_{+}\right\}$be a nonexpansive semigroup on $C, f$ be a bifunction from $C \times C$ to $\mathbb{R}$ satisfying conditions $\left(C_{1}\right)-\left(C_{4}\right)$. Suppose that $\Omega=\mathscr{F} \cap \operatorname{Sol}(f, C) \neq \varnothing$. Let $\left\{x_{n}\right\}$ and $\left\{u_{n}\right\}$ be sequences generated by

$$
\begin{aligned}
x^{0} & \in C \text { chosen arbitrarily, } \\
y_{n} & =\operatorname{argmin}\left\{\rho f\left(x_{n}, y\right)+\frac{1}{2}\left\|x_{n}-y\right\|^{2}: y \in C\right\}, \\
z_{n} & =\operatorname{argmin}\left\{\rho f\left(y_{n}, y\right)+\frac{1}{2}\left\|x_{n}-y\right\|^{2}: y \in C\right\}, \\
u_{n} & =\left(1-\mu_{n}\right) x_{n}+\mu_{n} T_{n} z_{n}, \\
H_{n} & =\left\{z \in \mathscr{H}:\left\|u_{n}-z\right\| \leq\left\|x_{n}-z\right\|\right\}, \\
W_{n} & =\left\{z \in \mathscr{H}:\left\langle x_{n}-z, x_{0}-x_{n}\right\rangle \geq 0\right\}, \\
x_{n+1} & =P_{\left(H_{n} \cap W_{n}\right)} x_{0},
\end{aligned}
$$


where $\left\{\mu_{n}\right\} \subset[a, 1], a \in(0,1)$. Then, $\left\{x_{n}\right\}$ and $\left\{u_{n}\right\}$ converge strongly to an element $p^{*} \in \Omega$.

Proof. Taking $N=1$ in Theorem 1, we get the desired conclusion easily.

Now putting $f_{i}(x, y)=0, i=1,2, \ldots, N$, for all $x, y \in C$, we obtain the following result for finding a common fixed point of a nonexpansive semigroup $\left\{T(s): s \in \mathbb{R}_{+}\right\}$ on $C$.

Corollary 2. Let $C$ be a nonempty closed convex subset in a real Hilbert space $\mathscr{H},\left\{T(s): s \in \mathbb{R}_{+}\right\}$be a nonexpansive semigroup on $C$ such that $\mathcal{F} \neq \varnothing$. Let $\left\{x_{n}\right\}$ and $\left\{u_{n}\right\}$ be sequences generated by

$$
\begin{aligned}
x_{0} & \in C \text { chosen arbitrarily, } \\
u_{n} & =\left(1-\mu_{n}\right) x_{n}+\mu_{n} T_{n} x_{n}, \\
H_{n} & =\left\{z \in \mathscr{H}:\left\|u_{n}-z\right\| \leq\left\|x_{n}-z\right\|\right\}, \\
W_{n} & =\left\{z \in \mathscr{H}:\left\langle x_{n}-z, x_{0}-x_{n}\right\rangle \geq 0\right\}, \\
x_{n+1} & =P_{\left(H_{n} \cap W_{n}\right)} x_{0},
\end{aligned}
$$

where $\left\{\mu_{n}\right\} \subset[a, 1], a \in(0,1)$. Then, $\left\{x_{n}\right\}$ and $\left\{u_{n}\right\}$ converge strongly to an element $p^{*} \in \mathcal{F}$.

If $T(s) x=x$ for all $s>0$ and $x \in C$, Algorithm 1 reduces to the following one for finding a common element in the solution-set of a finite family of pseudomonotone equilibrium problems in Hilbert spaces.

Corollary 3. Let $C$ be a nonempty closed convex subset in a real Hilbert space $\mathscr{H}, f_{i}$ be bifunctions from $C \times C$ to $\mathbb{R}$ satisfying conditions $\left(C_{1}\right)-\left(C_{4}\right)$. Suppose that $\Omega=\bigcap_{i=1}^{N} \operatorname{Sol}\left(f_{i}, C\right) \neq \varnothing$. Let $\left\{x_{n}\right\}$ and $\left\{u_{n}\right\}$ be sequences generated by

$x^{0} \in C$ chosen arbitrarily,

$$
\begin{aligned}
y_{n}^{i} & =\operatorname{argmin}\left\{\rho f_{i}\left(x_{n}, y\right)+\frac{1}{2}\left\|x_{n}-y\right\|^{2}: y \in C\right\}, i=1, \ldots, N, \\
z_{n}^{i} & =\operatorname{argmin}\left\{\rho f_{i}\left(y_{n}^{i}, y\right)+\frac{1}{2}\left\|x_{n}-y\right\|^{2}: y \in C\right\}, i=1, \ldots, N, \\
i_{n}= & \underset{1}{\operatorname{argmax}}\left\{\left\|z_{n}^{i}-x_{n}\right\|\right\}, \quad z_{n}:=z_{n}^{i_{n}}, \\
u_{n}= & \left(1-\mu_{n}\right) x_{n}+\mu_{n} z_{n}, \\
H_{n}= & \left\{z \in \mathcal{H}:\left\|u_{n}-z\right\| \leq\left\|x_{n}-z\right\|\right\}, \\
W_{n}= & \left\{z \in \mathcal{H}:\left\langle x_{n}-z, x_{0}-x_{n}\right\rangle \geq 0\right\}, \\
x_{n+1}= & P_{\left(H_{n} \cap W_{n}\right)} x_{0} .
\end{aligned}
$$

where $\left\{\mu_{n}\right\} \subset[a, 1]$ for some $a \in(0,1)$. Then, $\left\{x_{n}\right\}$ and $\left\{u_{n}\right\}$ converge strongly to an element $p \in \Omega$. 
To illustrate the proposed algorithm, we consider the following examples. The computer used in these experiments had an Intel Boxed Core CPU Q9400 6M Cache, $2.66 \mathrm{GHz}, 1333 \mathrm{MHz}$ FSB and $4 \mathrm{~GB}$ of memory. The language was MATLAB $2010 \mathrm{~b}$.

Example 1. Let $\mathscr{H}=\mathbb{R}^{k}$ with the inner product $\langle x, y\rangle:=x_{1} y_{1}+\cdots+x_{k} y_{k}$ for all $x=\left(x_{1}, x_{2}, \cdots, x_{k}\right), y=\left(y_{1}, y_{2}, \cdots, y_{k}\right) \in \mathscr{H}$. Let $C:=[-1,1]^{k}$ be a $k$-dimensional box in $\mathscr{H}$. For all $x, y \in C$ and for each $i \in\{1,2, \ldots, N\}$, we define the operator $f_{i}$ by

$$
f_{i}(x, y):=\sum_{j=1}^{k} \alpha_{i j}\left(y_{j}^{2}-x_{j}^{2}\right)
$$

where $\alpha_{i j} \in(0,1)$ are randomly generated. An elementary computation shows that conditions $\left(C_{1}\right)-\left(C_{4}\right)$ are satisfied for all $f_{i}, i=1,2, \ldots, N$. To define a nonexpansive semigroup let us consider the matrix

$$
T(s)=\left(\begin{array}{ccccc}
e^{-s} & 0 & 0 & \cdots & 0 \\
0 & e^{-s} & 0 & \cdots & 0 \\
0 & 0 & 1 & \cdots & 0 \\
\vdots & \vdots & \vdots & \ddots & \vdots \\
0 & 0 & 0 & \cdots & 1
\end{array}\right), s \in \mathbb{R},
$$

and let

$$
\begin{aligned}
T(s) x & =\left(\begin{array}{ccccc}
e^{-s} & 0 & 0 & \cdots & 0 \\
0 & e^{-s} & 0 & \cdots & 0 \\
0 & 0 & 1 & \cdots & 0 \\
\vdots & \vdots & \vdots & \ddots & \vdots \\
0 & 0 & 0 & \cdots & 1
\end{array}\right) x \\
& =\left(\begin{array}{ccccc}
e^{-s} & 0 & 0 & \cdots & 0 \\
0 & e^{-s} & 0 & \cdots & 0 \\
0 & 0 & 1 & \cdots & 0 \\
\vdots & \vdots & \vdots & \ddots & \vdots \\
0 & 0 & 0 & \cdots & 1
\end{array}\right)\left(\begin{array}{c}
x_{1} \\
x_{2} \\
\vdots \\
x_{k}
\end{array}\right) .
\end{aligned}
$$

It is easy to verify that $\{T(s): s \geq 0\}$ is a nonexpansive semigroup on $C$ and that the common solution-set is $\Omega=\mathcal{F} \cap\left(\bigcap_{i=1}^{N} \operatorname{Sol}\left(C, f_{i}\right)\right)=\left\{(0,0,0, \ldots, 0)^{T}\right\}$.

We apply Algorithm 1 to solve problem (1.1)-(1.2). Note that the mapping $T_{n}$ in Algorithm 1 can be found in a closed form:

$$
T_{n} z=\left(z_{1} \frac{1-e^{-s_{n}}}{s_{n}}, z_{2} \frac{1-e^{-s_{n}}}{s_{n}}, z_{3}, \ldots, z_{k}\right)^{T} .
$$

We choose the parameters as follows:

- $\rho=10$; 
- $\mu_{n}=0.9 \forall n \geq 1$

- $s_{n}=n \quad \forall n \geq 1$

- the stopping rule: $\left\|x_{n}-x^{*}\right\| \leq 5 \cdot 10^{-3}$, where $x^{*}=(0,0,0, \ldots, 0)^{T}$ is the unique solution of problem (1.1)-(1.2).

First, we test Algorithm 1 with $k=6, N=3, x_{0}=(1,1,1,1,1)^{T}$. The results are presented in Table 1 . The approximate solution is obtained after 198 iterations.

TABLE 1. Iterations of Algorithm 1 in Example 1 with starting point $x_{0}=(1,1,1,1,1,1)^{T}$

\begin{tabular}{lccccccc}
\hline Iter(n) & $x_{n}^{1}$ & $x_{n}^{2}$ & $x_{n}^{3}$ & $x_{n}^{4}$ & $x_{n}^{5}$ & $x_{n}^{6}$ & $\left\|x_{n}-x^{*}\right\|$ \\
\hline 0 & 1.0000 & 1.0000 & 1.0000 & 1.0000 & 1.0000 & 1.0000 & 2.4495 \\
1 & 0.5810 & 0.5720 & 0.6221 & 0.5845 & 0.5796 & 0.6328 & 1.4593 \\
2 & 0.3339 & 0.3348 & 0.3715 & 0.3507 & 0.3485 & 0.3787 & 0.8657 \\
3 & 0.2029 & 0.1925 & 0.2287 & 0.1993 & 0.1949 & 0.2360 & 0.5137 \\
4 & 0.0879 & 0.1278 & 0.1129 & 0.1499 & 0.1588 & 0.1103 & 0.3109 \\
5 & 0.3335 & 0.0252 & 0.2648 & -0.0727 & -0.1500 & 0.3029 & 0.5491 \\
6 & 0.1932 & 0.0471 & 0.1795 & -0.0013 & -0.0393 & 0.2039 & 0.3390 \\
7 & 0.1034 & 0.0715 & 0.1194 & 0.0610 & 0.0514 & 0.1309 & 0.2314 \\
$\cdots$ & $\cdots$ & $\cdots$ & $\cdots$ & $\cdots$ & $\cdots$ & $\cdots$ & $\cdots$ \\
198 & 0.0017 & 0.0017 & 0.0022 & 0.0017 & 0.0024 & 0.0023 & 0.0049 \\
\hline
\end{tabular}

Next, we test our algorithm with different choices of $k, N$ and $x_{0}$. The results are presented in Table 2.

TABLE 2. Performance of Algorithm 1 in Example 1 with different $k, N$ and $x_{0}$

\begin{tabular}{lccccc}
\hline & \multicolumn{3}{c}{$x_{0}=(1,1,1, \cdots, 1)^{T}$} & & \multicolumn{2}{c}{$x_{0}=(-1,-1,-1, \cdots,-1)^{T}$} \\
\cline { 2 - 3 } & CPU times & Iter. & & CPU times & Iter. \\
\hline $\mathrm{k}=6, \mathrm{~N}=3$ & 32.0823 & 135 & 32.7314 & 140 \\
$\mathrm{k}=6, \mathrm{~N}=6$ & 85.4715 & 171 & 110.8062 & 241 \\
$\mathrm{k}=10, \mathrm{~N}=6$ & 244.5807 & 423 & 231.3017 & 393 \\
$\mathrm{k}=20, \mathrm{~N}=3$ & 364.0974 & 889 & 351.2896 & 862 \\
\hline
\end{tabular}

Example 2. Consider problem (1.1)-(1.2) with $N=2$

$$
\text { find } x^{*} \in \Omega:=E P\left(C, f_{1}\right) \cap E P\left(C, f_{2}\right) \cap F \text {, }
$$

where

$$
f_{1}: \mathcal{R}^{3} \times \mathcal{R}^{3} \rightarrow \mathcal{R}, \quad f_{1}(x, y)=\|y\|^{4}-\|x\|^{4} \quad \forall x, y \in \mathcal{R}^{3},
$$




$$
\begin{gathered}
f_{2}: \mathcal{R}^{3} \times \mathcal{R}^{3} \rightarrow \mathcal{R}, f_{2}(x, y)=\langle A x+B y+q, y-x\rangle \forall x, y \in \mathcal{R}^{3}, \\
A=\left(\begin{array}{lll}
3 & 0 & 4 \\
2 & 6 & 3 \\
3 & 6 & 8
\end{array}\right), B=\left(\begin{array}{lll}
1 & 0 & 3 \\
2 & 3 & 4 \\
2 & 7 & 6
\end{array}\right), q=\left(\begin{array}{l}
2 \\
1 \\
1
\end{array}\right),
\end{gathered}
$$

$\mathrm{F}$ is the set of fixed points of the nonexpansive semigroup $T(t)$ defined by

$$
T(t): \mathcal{R}^{3} \rightarrow \mathcal{R}^{3}, T(t) x=\left(\begin{array}{ccc}
\cos t & -\sin t & 0 \\
\sin t & \cos t & 0 \\
0 & 0 & 1
\end{array}\right)\left(\begin{array}{l}
x_{1} \\
x_{2} \\
x_{3}
\end{array}\right), \forall x \in \mathcal{R}^{3}, t>0 .
$$

The feasible set is $C:=[0,1]^{3} \subset \mathcal{R}^{3}$. It is easy seen that $f_{1}, f_{2}$ is pseudomonotone and all the conditions of Theorem 1 are satisfied. Moreover, we can check that $\Omega=\left\{x^{*}\right\}$ where $x^{*}=(0,0,0)^{T}$ is the unique solution of problem (5.1). We apply Algorithm 1 to problem (5.1). Note that if we choose $s_{n}=n \quad \forall n \geq 1$, the mappings $T_{n}$ can be expressed in the form

$$
T_{n}(x)=\frac{1}{n}\left(\begin{array}{c}
x_{1} \sin n+x_{2}(\cos n-1) \\
x_{1}(1-\cos n)+x_{2} \sin n \\
n x_{3}
\end{array}\right) .
$$

Now, we compute the Lipschitz constants of $f_{i}$. It is easy seen that $f_{1}$ is Lipschitztype continuous with any constants $c_{1}, c_{2}>0$. For $f_{2}$, we have

$$
\begin{aligned}
f_{2}(x, y)+f_{2}(y, z)-f_{2}(x, z)= & \langle A x+B y+q, y-x\rangle+\langle A y+B z+q, z-y\rangle \\
& -\langle A x+B z+q, z-x\rangle \\
= & \langle A(y-x), z-y\rangle+\langle B(y-z), y-x\rangle \\
\geq & -\frac{(\|A\|+\|B\|)}{2}\|x-y\|^{2}-\frac{(\|A\|+\|B\|)}{2}\|y-z\|^{2} .
\end{aligned}
$$

Hence, $\frac{1}{2 c_{1}}=\frac{1}{2 c_{2}}=\frac{1}{\|A\|+\|B\|}=0.0418$. The parameters in Algorithm 1 are chosen as follows

- $\rho=0.04$

- $\mu=0.5$;

- $s_{n}=n \quad \forall n \geq 1$

- $x_{0}=(1,1,1)^{T}$;

- the stopping rule: $\left\|x_{n}-x^{*}\right\| \leq 5.10^{-3}$.

The results are presented in Table 3. The approximate solution is obtained after 137 iterations.

\section{CONCLUSION}

We have proposed a parallel iterative method for finding a common element in the solution sets of a finite family of pseudomonotone equilibrium problems and the set of fixed points for a semigroup nonexpansive mappings. For handling a finite family 
TABLE 3. Iterations of Algorithm 1 in Example 2.

\begin{tabular}{lcccc}
\hline Iter(n) & $x_{n}^{1}$ & $x_{n}^{2}$ & $x_{n}^{3}$ & $\left\|x_{n}-x^{*}\right\|$ \\
\hline 0 & 1.0000 & 1.0000 & 1.0000 & 1.7321 \\
1 & 0.8401 & 0.9462 & 0.8360 & 1.5166 \\
2 & 0.5758 & 0.8754 & 0.7388 & 1.2820 \\
3 & 0.4044 & 0.6031 & 0.6616 & 0.9823 \\
4 & 0.3552 & 0.3659 & 0.5562 & 0.7546 \\
5 & 0.2393 & 0.3172 & 0.4439 & 0.5957 \\
6 & 0.3476 & 0.1376 & 0.3436 & 0.5078 \\
7 & 0.1764 & 0.2634 & 0.3484 & 0.4711 \\
$\cdots$ & $\cdots$ & $\cdots$ & $\cdots$ & $\cdots$ \\
137 & -0.0020 & 0.0010 & 0.0010 & 0.0024 \\
\hline
\end{tabular}

of pseudomonotone equilibrium we have used a parallel splitting-up technique and the extragrandient with the Lipschitz-type continuous. The strong convergence of the proposed method has been established by using cutting planes.

\section{ACKNOWLEDGEMENT}

The research part of the first author was done during his visit to Center for Fundamental Science, Kaohsiung Medical University, Taiwan. The second author was partially supported by the grant MOST 106-2115-M-037-001 and the grant from Center for Nonlinear Analysis and Optimization, Kaohsiung Medical University, Taiwan. The third author was partially supported by the grant MOST 106-2923-E-039-001MY3.

\section{REFERENCES}

[1] M. Bianchi and S. Schaible, "Generalized monotone bifunctions and equilibrium problems," $J$. Optim. Theory Appl., vol. 90, no. 1, pp. 31-43, 1996, doi: 10.1007/BF02192244.

[2] E. Blum and W. Oettli, "From optimization and variational inequality to equilibrium problems ," Math. Stud., vol. 63, pp. 127-149, 1994.

[3] F. E. Browder, "Nonexpansive nonlinear operators in a Banach space ," Bull. Amer. Math. Soc., vol. 73, pp. 875-882, 1967, doi: 10.1090/S0002-9904-1967-11823-8.

[4] N. Buong, "Strong convergence of a method for variational inequality problems and fixed point problems of a nonexpansive semigroup in Hilbert spaces ," J. Appl. Math. Inform., vol. 20, pp. 61-74, 2011.

[5] L. C. Ceng and J. C. Yao, "Generalized monotone bifunctions and equilibrium problems," J. Optim. Theory Appl., vol. 90, pp. 31-43, 1996, doi: 10.1016/j.cam.2007.02.022.

[6] S. Chang, J. K. Kim, and L. Wang, "Total quasi- $\phi$-asymptotically nonexpansive semigroups and strong convergence theorems in Banach spaces ," Fixed Point Theory Appl., vol. 1, pp. 1-14, 2012, doi: 10.1186/1687-1812-2012-153. 
[7] P. L. Combettes, The convex feasibility problem in image recovery, In: Advances in Imaging and Electron Physics. New York: Academic Press, 1996.

[8] P. Daniele, F. Giannessi, and A. Maugeri, Equilibrium problems and variational models. New York: Springer, 2003.

[9] S. D. Flam and A. S. Antipin, "Equlibrium programming using proximal-like algorithms," Math. Prog., vol. 78, pp. 29-41, 1997, doi: 10.1007/BF02614504.

[10] K. Goebel and W. A. Kirk, Topics in Metric Fixed Point Theory of Cambridge Studies in Advanced Mathematics . Cambridge: Cambridge University Press, 1990.

[11] D. V. Hieu, L. D. Muu, and P. K. Anh, "Parallel hybrid extragradient methods for pseudomonotone equilibrium problems and nonexpansive mappings," Numer. Algorithm, vol. 73, pp. 197-217, 2016, doi: 10.1007/s11075-015-0092-5.

[12] Q. Jiang and J. Wang, "Hybrid algorithms of nonexpansive semigroup for mixed equilibrium problems, varitional inequalities and fixed point problems ," J. Inequal. Appl., vol. 174, 2014, doi: 10.1186/1029-242X.

[13] U. Kamraksa and R. Wangkeeree, "Generalized equilibrium problems and fixed point problems for nonexpansive semigroups in Hilbert spaces," J. Glob. Optim., vol. 51, pp. 689-714, 2011, doi: 10.1007/s10898-011-9654-9.

[14] S. Kitahara and W. Takahashi, "Image recovery by convex combinations of sunny nonexpansive retractions," Topol. Methods Nonlinear Anal., vol. 2, pp. 333-342, 1993.

[15] W. R. Mann, "Mean value methods in iteration," Proc. Amer. Math. Soc., vol. 4, pp. 506-510, 1953, doi: 10.1090/S0002-9939-1953-0054846-3.

[16] G. Mastroeni, On Auxiliary Principle for Equilibrium Problems. In: Daniele P., Giannessi F., Maugeri A. (eds) Equilibrium Problems and Variational Models. Nonconvex Optimization and Its Applications. Boston: Springer, 2003.

[17] N. Nadezhkina and W. Takahashi, "Weak convergence theorem by an extragradient method for nonexpansive mappings and monotone mappings," J. Optim. Theory Appl., vol. 128, pp. 191-201, 2006, doi: 10.1007/s10957-005-7564-Z.

[18] M. A. Noor, "General variational inequalities and nonexpansive mappings," J. Math. Anal. Appl., vol. 331, pp. 810-822, 2007, doi: 10.1016/j.jmaa.2006.09.039.

[19] Z. Opial, "Weak convergence of the sequence of successive approximations for nonexpansive mappings," Bull. Amer. Math. Soc., vol. 73, pp. 591-597, 1967, doi: 10.1090/S0002-9904-196711761-0.

[20] T. D. Quoc, L. D. Muu, and N. V. Hien, "Extragradient algorithms extended to equilibrium problems," Optimization, vol. 57, pp. 749-776, 2008, doi: 10.1080/02331930601122876.

[21] S. Saeidi, "Iterative algorithms for finding common solutions of variational inequalities and systems of equilibrium problems and fixed points of families and semigroups of nonexpansive mappings," Nonlinear Anal., vol. 70, pp. 4195-4208, 2009, doi: 10.1016/j.na.2008.09.009.

[22] Y. Shehu, "An iterative method for nonexpansive semigroup, variational inclusions and generalized equilibrium problems," Math. Comput. Modelling, vol. 55, pp. 1301-1314, 2012, doi: 10.1016/j.mcm.2011.10.008.

[23] T. Shimizu and W. Takahashi, "Strong convergence to common fixed points of families of nonexpansive mappings," J. Math. Anal. Appl., vol. 211, pp. 71-83, 1997, doi: 10.1006/jmaa.1997.5398.

[24] T. Suzuki, "Characterrizations of common fixed points of one-parameter nonexpansive semigroups, and convergence theorems to common fixed points," J. Math. Anal. Appl., vol. 324, pp. 1006-1019, 2006, doi: 10.1016/j.jmaa.2006.01.004.

[25] Y. Yao and J. C. Yao, "On modified iterative method for nonexpansive mappings and monotone mappings," Appl. Math. Comput., vol. 186, pp. 1551-1558, 2007, doi: 10.1016/j.amc.2006.08.062. 


\section{Authors' addresses}

\section{L.Q. Thuy}

School of Applied Mathematics and Informatics, Hanoi University of Science and Technology, No 1, Dai Co Viet Road, Hai Ba Trung, Hanoi, Vietnam. Center for Fundamental Science, Kaohsiung Medical University, Kaohsiung 80708, Taiwan.

E-mail address: thuy. lequang@hust.edu.vn

\section{C.-F. Wen}

Center for Fundamental Science, and Research Center for Nonlinear Analysis and Optimization, Kaohsiung Medical University, Kaohsiung 80708, Taiwan. Department of Medical Research, Kaohsiung Medical University Hospital, Kaohsiung, 80708, Taiwan.

E-mail address: cfwen@kmu.edu.tw

\section{J.-C. Yao}

Center for General Education, China Medical University, Taichung 40402, Taiwan. Research Center for Interneural Computing, China Medical University Hospital, Taichung 40447, Taiwan.

E-mail address: yaojcemail.cmu.edu.tw

\section{T. N. Hai}

School of Applied Mathematics and Informatics, Hanoi University of Science and Technology, No 1, Dai Co Viet Road, Hai Ba Trung, Hanoi, Vietnam.

E-mail address: hai.trinhngoc@hust.edu.vn 